\title{
Projetos de aprendizagem como estratégia de ensino de informática para pessoas com deficiência intelectual: Estudo de caso
}

\section{Learning projects as a computer teaching strategy for people with intellectual disabilities: a case study}

\author{
Paulo Antonio Pasqual Júnior ${ }^{1}$ \\ ${ }^{1}$ Universidade de Caxias do Sul - UCS, Caxias do Sul, RS, Brasil
}

Autor para correspondência/Mail to: Paulo Antonio Pasqual Júnior (papjunior@ucs.br)

Recebido/Submitted: 29 Jul. 2017; Aceito/Approved: 22 Set. 2017

Copyright (C) 2017 Pasqual Júnior, P. A.. Todo o conteúdo da Revista (incluindo-se instruções, política editorial e modelos) está sob uma licença Creative Commons Atribuição-NãoComercial-Compartilhalgual 3.0 Não Adaptada. Ao serem publicados por esta Revista, os artigos são de livre uso em ambientes educacionais, de pesquisa e não comerciais, com atribuição de autoria obrigatória. Mais informações em http://revistas.ufpr.br/atoz/about/submissions\#copyrightNotice.

\begin{abstract}
Resumo
Introdução: As tecnologias têm transformado a maneira como as pessoas vivem e se relacionam com o conhecimento, trazendo subsídios para a potencialização de processos de ensino-aprendizagem. Uma alternativa, nesse sentido são os projetos de aprendizagem apoiados em computadores, os quais permitem ao aluno a construção do seu próprio conhecimento. Essas metodologias vêm sendo usadas em várias áreas da educação, sendo que o objetivo deste artigo é apresentar um relato de experiência sobre um projeto de aprendizagem em informática de um curso de Auxiliar de Serviços voltado a pessoas com deficiência intelectual.

Método: consistiu em um estudo de caso de natureza qualitativa.

Resultados: O projeto envolveu alunos com deficiência intelectual, o monitor e o professor, evidenciando-se que a aplicação da metodologia de projetos de aprendizagem resulta em um recurso eficiente para o ensino de informática para pessoas com deficiência intelectual.

Conclusões: As evidências apontam que os projetos de aprendizagem podem ser uma alternativa eficaz para o trabalho com pessoas com deficiência intelectual.
\end{abstract}

Palavras-chave: Projetos de aprendizagem; Deficiência intelectual; Informática em educação

\begin{abstract}
Introduction: Technologies have transformed the way people live and relate to knowledge, bringing the potential for teaching-learning processes. An alternative in this sense is the learning projects supported by computers, which allow the student to build their own knowledge. These methodologies have been used in several areas of education, and the purpose of this article is to present an experience report about a learning project focused on computer learning in a Service Assistant course for people with intellectual disabilities.

Method: consisted of a qualitative case study.

Results: The project involved students with intellectual disability, monitor and teacher, demonstrating that the application of the methodology of learning projects results in an efficient resource for the teaching of information technology for people with intellectual disabilities.

Conclusions: Evidence suggests that learning projects can be an effective alternative for working with people with intellectual disabilities.

Keywords: Learning projects; intellectual impairment; Informatics in education
\end{abstract}

\section{INTRODUÇÃO}

A utilização da informática como suporte à aprendizagem tem sido uma prática comum em todas as modalidades de ensino, inclusive no campo da educação especial. Segundo Papert (2008) o computador é um importante recurso de aprendizagem, pois a criança, a partir da interação com o computador, é capaz de construir conhecimentos que vão além dos apresentados pelos métodos tradicionais.

Para Pan (2008) a pessoa com deficiência intelectual não é um estereótipo de incapacidades, mas sim alguém que apresenta uma forma particular de pensamento e está aberta para seu desenvolvimento dentro de sua capacidade específica. Nesse sentido, o conceito de deficiência, tende a ser desvinculado das quantificações de coeficiente intelectual (QI), possibilitando um olhar a partir das capacidades e potencialidades de cada indivíduo.

O ensino de pessoas com deficiência intelectual por muito tempo esteve vinculado a metodologias empiristas, que fomentavam a repetição para a aprendizagem. Glat e Pletsch (2004) apontam para a necessidade de deslocar as intervenções do foco nas dificuldades e carências para alternativas de educação com foco preferencial nas ações interdisciplinares e nos potenciais do estudante com deficiência intelectual. Valentini, Gomes, e Bisol (2016) apontam como aspectos importantes do sucesso nos processos de ensino e aprendizagem do estudante com deficiência intelectual, o fortalecimento das habilidades sociais e da interação entre colegas - a partir do desenvolvimento de práticas educativas - e programas que promovam novas interações no grupo e que fortaleçam os vínculos entre os estudantes. Nessa perspectiva, projetos de aprendizagem podem ser uma 
alternativa para possibilitar o crescimento da autonomia e a possibilidade de construção do conhecimento em pessoas com deficiência intelectual. Para Fagundes, Sato, e Maçada (1999), um projeto de aprendizagem consiste em permitir ao aluno a escolha de um tema de pesquisa, para que a partir da motivação do aluno ele possa construir conhecimentos. Nas palavras dos autores, “[q]uando falamos em 'aprendizagem por projetos' estamos necessariamente nos referindo à formulação de questões pelo autor do projeto, pelo sujeito que vai construir conhecimento" (Fagundes et al., 1999, p. 16).

Nesse sentido, o objetivo deste artigo é apresentar os resultados da utilização de projetos de aprendizagem como metodologia para o ensino-aprendizagem de informática, em uma turma de pessoas com deficiência intelectual, participantes de um projeto Menor Aprendiz.

\section{PROCEDIMENTOS METODOLÓGICOS E RESULTADOS}

O programa “Menor Aprendiz” é uma política pública instituída pelo governo federal que tem por objetivo oportunizar o ingresso de jovens no mercado de trabalho. Nessa direção, a execução da Lei № 8.213 (Brasil, 1991) tem possibilitado a inúmeros jovens e adultos com diversas deficiências a inclusão no mercado de trabalho. No caminho dessas mudanças, e aliado às demandas da sociedade, nasceu o curso de Auxiliar de Serviços para pessoas com Deficiência Intelectual, com o objetivo de possibilitar uma formação que permitisse aos jovens a inclusão no mercado de trabalho. Esse curso se configurou com duração de oito meses, mesclando atividades em sala de aula e experiências no ambiente de trabalho, ao qual o jovem aprendiz estava vinculado. $\mathrm{O}$ curso foi oferecido aos jovens da comunidade por uma instituição de ensino superior de uma cidade da Serra Gaúcha em parceria com os hospitais da rede pública e privada da mesma Região. Esta iniciativa se diferenciou de outras já realizadas por instituições como o Serviço Nacional de Aprendizagem Comercial (SENAC), em especial por se tratar de um curso exclusivo para pessoas com deficiência intelectual e que contemplou atividades que vão além dos saberes exclusivamente técnicos. Dentre as disciplinas de formação do curso estavam presentes: Leitura e Escrita, Matemática, Atividades da Vida Diária, Corporeidade e Informática. O estudo aqui apresentado foi desenvolvido na disciplina de Informática com uma turma composta por catorze alunos maiores de dezoito anos de diversas idades, e envolveu múltiplos diagnósticos de deficiência intelectual. Destes, apenas dois possuíam diagnóstico de Síndrome de Down e o restante não possuíam classificação definida pelo Sistema Internacional de Doenças (SID). Atualmente a Deficiência Intelectual é definida por uma incapacidade "caracterizada por limitações significativas no funcionamento intelectual e no comportamento adaptativo, como expresso nas habilidades práticas, sociais e conceituais, originando-se antes dos dezoito anos de idade" (Luckasson et al., 2002, p. 08).

Desse modo, este estudo buscou responder a seguinte questão investigativa: Como pessoas com deficiência intelectual podem aprender informática por meio de projetos de aprendizagem?

A metodologia utilizada para este trabalho caracterizou-se como um estudo de caso de natureza qualitativa, tendo como fontes de evidência os registros das interações dos alunos no diário de campo do pesquisador, as manifestações em um grupo privado online e as produções desenvolvidas pelos alunos. De acordo com Yin (2010) o estudo de caso consiste em:

Uma investigação empírica que investiga um fenômeno contemporâneo em profundidade e em seu contexto de vida real, especialmente quando os limites entre o fenômeno e o contexto não são claramente evidentes. (p.39)

A escolha deste curso para a realização deste estudo se deu pelo ineditismo da proposta, em especial da instituição em propor um curso em modalidade de "Menor Aprendiz" exclusivo para pessoas com deficiência intelectual. Em segundo lugar, por sabermos que, de maneira geral, os recursos de instrução de pessoas com deficiência são extensivamente pautados em pedagogias de cunho comportamentalista.

Assim, o projeto intitulado "Ser pesquisador”, envolveu assuntos à escolha dos alunos. Eles foram convidados a trabalhar em grupos e interagirem de maneira construtiva tendo a finalidade de potencializar a aprendizagem nos mais variados níveis, não apenas no que dizia respeito aos conteúdos conceituais de informática, mas também em relação à socialização, ao respeito ao outro e ao desenvolvimento da competência de saber trabalhar em grupo, preparando-os principalmente para o mundo do trabalho.

Dessa forma o principal objetivo deste projeto de aprendizagem foi possibilitar aos alunos a capacidade de aprender a aprender utilizando os recursos do computador. A intenção primeira não foi especificamente que os mesmos aprendessem a editar imagem ou vídeo, por exemplo, mas torná-los pesquisadores, e desenvolverem seus próprios métodos de aprendizagem com base no que o computador pode oferecer aos usuários.

Como meio de registro das atividades, criou-se um grupo do Facebook, utilizado como ambiente virtual de aprendizagem. Se por um lado o Facebook pode ser um recurso de dispersão e, muitas vezes de problemas em sala de aula, por outro, pode ser uma poderosa ferramenta de socialização e construção do conhecimento. Assim, a utilização de um grupo possibilitou discussões e compartilhamentos, significativos para todos os envolvidos no projeto. Nesse sentido, cabe salientar o estudo de Bernardi e Silva (2015), em que apresentam as 
inúmeras possibilidades do uso do Facebook, como recurso de construção do conhecimento em vários estágios da educação.

É importante salientar que o projeto esteve embasado no modelo teórico proposto por Fagundes et al. (1999), em que um projeto de aprendizagem se constitui em atividades nas quais o aluno é protagonista. Em outras palavras, as atividades são desenvolvidas a partir dos questionamentos, anseios e motivação dos alunos na busca pelo conhecimento. Nesse sentido, as etapas do projeto se constituíram a partir de um modelo criado pelo professor: Etapa 1 - técnica de brainstorm; Etapa 2 - criação dos grupos; Etapa 3 - desenvolvimento das atividades propostas; e Etapa 4 - apresentação das atividades ao grande grupo.

Na primeira etapa, os alunos, professor e monitor conversaram sobre os anseios de aprendizado do grupo trazendo à tona questionamentos e ideias para serem trabalhadas a partir de uma atividade direcionada de brainstorm. Em alguns minutos foram citados os seguintes assuntos: Photoshop, confecção de vídeos, edição de músicas, Excel e vírus de computador. A partir dessas ideias, elencaram-se os seguintes assuntos principais: Edição de Imagens, Edição de Vídeo, Manipulação de Dados em Planilhas Eletrônicas, e Segurança de Dados. Os assuntos foram adaptados em virtude das licenças necessárias para se trabalhar com alguns dos softwares citados. Além disso, o professor procurou escolher softwares que fossem suficientemente intuitivos para que os alunos pudessem desenvolver as atividades de exploração e construção de maneira autônoma.

Assim, os alunos foram agrupados tendo como critério exclusivo os assuntos manifestados na etapa anterior, formando-se quatro grupos (A, B, C e D) orientados a desenvolver um cronograma de trabalho para cinco aulas. O objetivo da confecção do cronograma foi possibilitar habilidades de planejamento aos alunos; e que estes desenvolvessem a competência de tomar decisões. Desse modo, seria então possível perceber posteriores necessidades como, por exemplo, reconhecer a importância de ter objetivos, tanto na vida escolar como profissional ou pessoal.

Considerando-se que "[é] fundamental que a questão a ser pesquisada parta da curiosidade, das dúvidas, das indagações do aluno, ou dos alunos, e não imposta pelo professor. Isto porque a motivação é intrínseca, é própria do indivíduo" (Fagundes et al., 1999, p. 16), a opinião dos alunos e seus questionamentos e anseios acerca do que não tinham ainda estudado foi imprescindível para o bom andamento da aprendizagem. Juntamente com o professor, todas as decisões foram tomadas, de maneira que cada indivíduo participasse democraticamente do processo de aprendizagem.

Nesta etapa retomou-se a atividade, ficando acordado que ao término de cada aula os alunos postariam uma síntese do que foi trabalhado no ambiente virtual. Quando o professor era questionado respondia com outras perguntas, tendo como objetivo promover um processo de metacognição. Nesse sentido, o papel do professor conforme Silva (2004) passa de apenas transmissor para sistematizador de experiências. Assim, o professor passou a questionar e sistematizar problemas e situações que possibilitassem uma ressignificação de saberes e consequentemente a construção do conhecimento. Para Fagundes et al. (1999) o projeto de aprendizagem se constitui pela autonomia na escolha do que deve ser pesquisado e pela ação reflexiva entre todas as partes envolvidas dentro do processo propriamente dito. Dessa forma, os alunos e professor reuniam-se constantemente para discutir e tomar decisões coletivas com relação ao andamento do projeto.

Em sequência ao projeto, a Quarta Etapa constituiu-se das apresentações interativas, ou seja, os alunos não apenas apresentavam seus trabalhos, mas levavam a turma toda a realizar as atividades propostas por eles.

Em geral, as apresentações de trabalho foram semelhantes: enquanto um ou dois alunos, à escolha do grupo, faziam a apresentação do tema e davam instruções de como realizar a atividade prática, a outra parte do grupo se preocupava em auxiliar os colegas no desenvolvimento do trabalho proposto. Os grupos A e B apresentaram de forma organizada e conseguiram levar os colegas a desenvolverem as atividades propostas. Uma diferença entre os dois grupos, contudo, foi observada quanto à autonomia e organização, uma vez que o primeiro necessitou de menos intervenções, comparado ao segundo. Os grupos C e D apresentaram maiores dificuldades na execução das tarefas, em especial por terem uma composição mais heterogênea em relação aos dois primeiros. É importante ressaltar que o grupo $\mathrm{C}$ apresentou dificuldades na execução de parte significativa das tarefas necessitando intervenções mais direcionadas, se comparadas às atividades desenvolvidas pelos outros grupos. Foi necessário, por exemplo, solicitar que os alunos escrevessem frases de até duas linhas para evitar o "copiar e colar”. Em especial essas dificuldades se apresentaram em virtude de dificuldades da maior parte do grupo tanto com questões de linguagem escrita como com questões de letramento digital. Ainda assim, ambos os grupos evidenciaram aprendizagem e comprometimento com as atividades propostas evidenciando que projetos de aprendizagem podem ser utilizados com pessoas com deficiência intelectual.

\section{CONCLUSÕES}

Há barreiras consideráveis a vencer quando se fala em educação, ainda mais no que tange a educação de pessoas com deficiência. Porém, percebe-se importante potencial a ser explorado nesse campo. 
Com este estudo percebeu-se que, no decorrer das aulas, muitas vezes, havia resistência da turma em fazer escolhas e desenvolver o pensamento livremente, o que levou o grupo de pesquisadores a presumir que tal atitude se baseia no fato de abordagens diretivas serem práticas comuns no ensino-aprendizagem de pessoas com deficiência intelectual. Entretanto, com o passar do tempo, através das interações, trocas, discussões e escolhas, o grupo passou a perceber as suas próprias potencialidades e ao final do projeto conseguiu desenvolver trabalhos com significado e qualidade.

Se por um lado este estudo de caso apresenta uma possibilidade metodológica para ensino-aprendizagem de pessoas com deficiência intelectual, por outro, permite que outras questões sejam levantadas para estudos futuros, tais como: Como os cursos de formação em modalidade "menor aprendiz" para pessoas com deficiência têm se configurado no Brasil? Que metodologias para o ensino-aprendizagem têm sido contempladas nesses cursos? Como projetos de aprendizagem podem ser utilizados como metodologia para o ensino de pessoas com outras deficiências? Nesse sentido, esses questionamentos possibilitam pensar em uma modalidade de ensino que perpasse a transmissão de conhecimentos.

Há trabalhos que apontam as dificuldades e potencialidades da inclusão de pessoas com deficiência, tanto na escola (Valentini et al., 2016) como no mundo do trabalho (Ribeiro, Batista, Prado, Vieira, \& Carvalho, 2014). Contudo, neste momento do estudo não se localizou, na literatura, discussões acerca de projetos de aprendizagem como alternativa para o ensino de pessoas com deficiência, tampouco trabalhos que tratem do ensino de informática para pessoas com deficiência intelectual. No campo da informática, contudo, os estudos presentes na literatura versam geralmente sobre o uso de softwares inclusivos e de tecnologias assistidas, as quais possibilitam a inclusão de pessoas com deficiência intelectual, mas não discutem as potencialidades e a necessidade do aprendizado de informática aliado ao mundo do trabalho.

Nesse sentido, é importante ressaltar a relevância desse estudo e a necessidade de novas pesquisas e discussões que possibilitem outros caminhos acerca do ensino-aprendizagem de pessoas com deficiência intelectual. É importante reconhecer, no entanto, a limitação deste estudo, uma vez que não é passível de generalização, em especial por se tratar de um estudo de natureza qualitativa. Entretanto, apresenta-se uma possibilidade para se pensar o uso de projetos de aprendizagem como um recurso para o ensino de informática para pessoas com deficiência intelectual a partir de um projeto realizado em um curso de Menor Aprendiz. 


\section{REFERÊNCIAS}

Bernardi, P. M., \& Silva, S. A. (2015). O uso do Facebook como ferramenta para o compartilhamento de atividades pedagógicas: uma experiência em uma escola pública de educação infantil. AtoZ: novas práticas em informação e conhecimento, 4(2), 108 - 112. doi: 10.5380/atoz.v4i2.43496

Brasil. (1991). Lei n ${ }^{0} 8.213$, de 24 de julho de 1991. Presidência da República do Brasil. Recuperado em 13 de outubro de 2017, de http://www.planalto.gov.br/ccivil_03/leis/ L8213cons.htm

Fagundes, L. D. C., Sato, L. S., \& Maçada, D. L. (1999). Aprendizes do futuro: as inovações começaram! Recuperado em 2 mar. 2017, de http://escola2000.net/futura/textos-proinfo/ livro03Lea\%20Fagundes\%20et\%20alii.pdf

Glat, R., \& Pletsch, M. D. (2004). Orientação familiar como estratégia facilitadora do desenvolvimento e inclusão de pessoas com necessidades especiais. Revista Educação Especial(24). doi: 10.5902/1984686X

Luckasson, R., Borthwick-Duffy, S., Buntinx, W. H. E., Coulter, D. L., Craig, E. M., Reeve, A., ... Tassé, M. J. (2002). Mental retardation: definition, classification, and systems of support. Washington, DC: American Association on Mental Retardation.

Pan, M. A. G. S. (2008). O direito à diferença: uma reflexão sobre deficiência intelectual e educação inclusiva. Curitiba: IBEPEX.

Papert, S. (2008). A máquina das crianças: repensando a escola na era da informática. Porto Alegre: Artmed.

Ribeiro, A. P., Batista, D. F., Prado, J. M., Vieira, K. E., \& Carvalho, R. L. (2014, ago./dez.). Cenário da inserção de pessoas com deficiência no mercado de trabalho: revisão sistemática. Revista da Universidade Vale do Rio Verde, 12(2), 268 - 276. doi: 10.5892/ruvrd.v12i2.1441

Silva, M. (2004). Indicadores de interatividade para o professor presencial e on-line. Revista Diálogo Educacional, 4(12). Recuperado de http://www2.pucpr.br/reol/index.php/ dialogo dd $1=622 \& d d 2=670 \& d d 3=\& d d 99=$ pdf

Valentini, C. B., Gomes, R. B., \& Bisol, C. A. (2016). Inclusão de estudantes com deficiência intelectual: uma revisão sistemática da literatura. Revista Teias, 17, 125-142. doi: 10.12957/teias.2016.25502

Yin, R. K. (2010). Estudo de caso: planejamento e métodos (4a. ed.). Porto Alegre: Bookman.

Como citar este shortpaper (APA):

Pasqual Júnior, P. A. (2017). Projetos de aprendizagem como estratégia de ensino de informática para pessoas com deficiência intelectual: Estudo de caso. AtoZ: novas práticas em informação e conhecimento, 6(1), 26 - 30. Recuperado de: http://dx.doi.org/10.5380/atoz.v6i1 .54196 